\begin{tabular}{|l|l|l|l|l|}
\hline Revista Clío América & ISSN: 1909-941X & Vol. 8 & No. 15 & Enero - Junio de 2014 \\
\hline
\end{tabular}

\title{
La cultura organizacional de los operadores turísticos de Santa Marta 2012-2013
}

\author{
The organizational culture of Santa Marta tour operators 2012-2013
}

\begin{abstract}
Resumen: Con base en la inquietud por desarrollar estrategias que conlleven al aumento en la productividad de los operadores turísticos de la ciudad de Santa Marta, esta investigación se propone caracterizar las condiciones de la cultura organizacional de dichas instituciones; en este estudio, se definen las variables correspondientes a las condiciones físicas de trabajo, las del entorno laboral y el comportamiento organizacional, como elementos de la cultura que al ser estudiados, tienen como fin establecer su relación con el rendimiento laboral de los empleados en las empresas turísticas. Para el estudio en Santa Marta, se determinaron las características de cada variable en un cuestionario de 24 preguntas, se encuestaron miembros de las organizaciones y se ahondó en la percepción de directivos, administrativos y operativos, e identificar para el caso de la ciudad, si el talento humano se encuentra motivado en gran parte por las relaciones interpersonales, las condiciones físicas de trabajo y un entorno laboral adecuado para el desarrollo de su trabajo, y así observar la relación frente a su rendimiento.
\end{abstract}

Palabras Clave: Cultura organizacional, condiciones de trabajo, entorno laboral, comportamiento organizacional.

JEL: M120

\begin{abstract}
Based on the concern to develop strategies that lead to increased productivity of tourism operators in the city of Santa Marta, this research aims to characterize the condition of the organizational culture of these institutions, in this study, the corresponding variables are defined the physical conditions of work, work environment conditions and organizational behavior, as elements of the culture being studied, are intended to establish its relationship with the work performance of employees in tourism enterprises. For the study in Santa Marta, the characteristics of each variable were determined on a questionnaire of 24 questions, were surveyed the members of the organizations deepening in the perception of managers, administrative and operational, and identify for the case of the city, if human talent is largely motivated by interpersonal relations, physical working conditions and an adequate working environment for the development of their work, and observe the relationship in front of his performance.
\end{abstract}

Keywords: Organizational culture, working conditions, work environment, organizational behavior.
Jennifer Tatiana Ortiz Segrera

VII semestre de Administración de Empresa Universidad del Magdalena, Colombia jenny.segrera.9426@gmail.com

Alexander Daza Corredor Maestria en Administracion Universidad del Magdalena, Colombia alexdaza71@hotmail.com

Carlos Labarcés Ballestas Maestria en Administracion Universidad del Magdalena, Colombia clabarces@hotmail.com

Tipología:

Artículo de Investigación Científica Fecha de Recibido: Diciembre 09 de 2013 Fecha de Aceptación: Marzo 10 de 2014

Para citar este artículo: Ortíz, S. J., Daza, C. A., \& Labarcés, B. C. (2014). La cultura organizacional de los operadores turísticos de Santa Marta 2012 - 2013. Clío América, 8 (15), 22 - 35 


\section{Introducción}

La teoría administrativa con el paso del tiempo se ha actualizado acorde con las nuevas propuestas de trabajo que han surgido en las organizaciones, si se tiene en cuenta que el ambiente laboral determina si es para un trabajador agradable o no desempeñar su labor con efectividad; es notable destacar, entonces, que es en el talento humano en donde se encuentran las principales fortalezas y debilidades de las organizaciones, en todos los procesos como los productivos, administrativos y de servicio. (De la Cruz \& Hernández, 2007, p. 23; Kahya, 2009, p.98). Entendemos lo estratégico de un buen ambiente laboral en la organización, que se cohesione con las necesidades e intereses de su talento humano para el logro de los objetivos organizacionales, en la actualidad, ese es el objetivo de todo gerente.

Al revisar la literatura, es innegable encontrar cómo los conceptos de diversos autores se retroalimentan y fortalecen a través del tiempo, parafraseando a Guerrero \& Navarro (2003, p.27) y Morales (2010, p.11) quienes en su definición realizada con base en aportes de diversos autores, sostienen que la cultura organizacional es la conciencia colectiva 0 un conjunto de formas de pensar y sentir que se expresan mediante sistemas de significados compartidos por los miembros de la organización que los identifica y diferencia de otros por sus características, institucionalizando así sus conductas sociales y convirtiéndola en una colectividad particular y distinta. Aquí empezamos a interiorizar la cultura organizacional y su influencia en las relaciones y productividad de sus integrantes, entendiendo que esta es propia de cada organización y dista de otras.

Al referirse a la cultura organizacional como la utilización y apropiación de un conjunto de valores creados dentro de la misma para provecho en el desarrollo de su trabajo, Martín (1992) y Hofstede (1991) (citados por Calderón \& Serna, 2009) analizando este elemento inherente a toda organización determinan que para las organizaciones la cultura toma dos enfoques y los presenta de la siguiente manera:

Un grupo afirma que la cultura es 'algo' que la organización es y por lo tanto no puede ser in- tervenible intencionalmente. Otro grupo, por el contrario, considera que la cultura es 'algo' que la organización tiene y en este sentido podría ser intervenida en busca de mejores resultados organizacionales. (p.98)

Puede entenderse la cultura organizacional como un instrumento que hace más fácil la consecución de los objetivos, y si es capaz de generar valor a la organización, entonces, su cultura será distinta a la de cualquier otra organización y, por ende, será difícil de imitar; lo que se convertiría en un activo estratégico y una ventaja competitiva que direcciona el rumbo de la compañía; ya que comparten historia, normatividad y objetivos comunes trazados como colectividad, asumiéndolos también como objetivos propios (Tavares, 2004, p.69; González \& Parra, 2008, p.46; Gálvez \& García, 2011, p.129). Estas posiciones teóricas, ratifican la postura que tienen los autores, en el sentido de entender la cultura organizacional como algo propio e intangible de cada organización, difícil de imitar, pero influenciable de cierto modo en el sentido de articular y direccionar los interés de la organización a la de sus empleados, lo que se traduce en valor agregado para la organización.

El presente documento se centrará en describir la relación que se presenta entre las variables de las condiciones físicas de trabajo, el entorno laboral y la comunicación, y el comportamiento organizacional, con el fin de determinar la relación que presentan con el rendimiento laboral y la consecución de logros organizacionales en los principales hoteles, restaurantes y agencias de viajes de la ciudad de Santa Marta.

\section{Condiciones de Trabajo}

Las condiciones físicas y ambientales, es uno de los más importantes factores a los cuales se expone el trabajador en el desarrollo de sus labores, tales como el calor, la humedad, el ruido, el olor, entre otros; los cuales tienen efectos directos e indirectos sobre el desempeño laboral de éstos. La concentración en las tareas de un empleado que se expone a estos impactos disminuye, creando un estrés emocional que conduce en último término a un bajo rendimiento en la productividad y la calidad (Kahya, 2007, p.517). 
Un trabajador espera una retribución equilibrada con los esfuerzos que ellos realizan en las organizaciones, es por esto que requieren de condiciones apropiadas para desempeñarse de la mejor manera (Tziner \& Sharoni, 2014, p.36). A mayor esfuerzo y exposición a condiciones físicas y ambientales adversas, se aspira a una compensación mayor, lo que redunda en la motivación del empleado. Las condiciones de trabajo no solo incluyen los aspectos de distribución del espacio de trabajo, y los implementos adecuados para la realización del mismo; es necesario también contemplar la necesidad de seguridad de la cual hablan repetidamente distintos autores que han delimitado la teoría organizacional, tales como Maslow, Fayol, Taylor y Herzberg, entre otros; e igualmente los factores ambientales (Kahya, 2007, p.517).

En efecto, el desempeño de un trabajador depende en gran medida de la adecuación de un ambiente de trabajo favorable, es así como se habla de las recompensas del clima y la cultura, dado que los trabajadores esperan que al desempeñarse en su trabajo se realicen también sus objetivos individuales creando así lazos psicológicos con la organización y los beneficios que ésta le ofrece en su desarrollo personal, generando un efecto directo en la capacidad productiva e innovadora del equipo; ésta depende en gran parte de los valores culturales de la organización y los riesgos que las personas consideran aceptables asumir al emprender un mayor desempeño dentro de su actividad productiva. Las definiciones de diversos autores sustentan esta posición afirmando que muchos trabajadores aceptan la responsabilidad de producir a un alto nivel cuando están organizados eficazmente en su trabajo (Chiang \& Birtch, 2011, p.4; Turró, Urbano $\&$ Peris, 2013, p.4). Se reafirma la postura que las condiciones laborales influyen en la mayor capacidad productiva de los empleados y generación de valor para la organización.

\section{Relaciones Laborales}

Los aportes de las investigaciones como la de Doherty \& MacIntosh (2009, p.108) y Méndez (2006, p.17) permiten extraer algunos factores que determinan las condiciones culturales en las cuales se desenvuelven los trabajadores diariamente, tales como: Los canales de comunicación con administrativos y directivos, la formalización, las ventas, la competencia del personal, el ambiente, entre otros; dichos factores influyen en el nivel de satisfacción de los trabajadores con el desempeño de su trabajo.

Tsui, Zhang, Wang, Xin \& Wu. (2006, p.117), hacen referencia a la relevancia de las relaciones de trabajo al expresar en su concepto, que la cultura de una empresa nace del trabajo en equipo y no por imposición normativa, y de esto se desprende el éxito y compromiso de los trabajadores con la labor que desempeñan; así mismo, lo muestran Açıkgöz \& Günsel (2011) cuando exponen que: “Un apoyo abierto y compartido de la visión innovadora, de la percepción y el estímulo de la alta dirección hacia objetivos innovadores aumentan significativamente la capacidad de innovación a nivel de equipo". (p.922). Se observa que las relaciones interpersonales adecuadas en todos los niveles, son un elemento clave en la generación de sinergias en los equipos de trabajo y a su capacidad de creación; lo importante de ésto, es que genere valor para la organización y logre articularse con las necesidades y expectativas de sus empleados.

Lo anterior, se complementa con la posición de Jarratt \& O'Neill (2002) al afirmar que: "La cultura organizativa y sus valores subyacentes, influye en el comportamiento y las expectativas individuales de los administradores dentro de una empresa". (p.22)

La investigación buscó identificar de igual forma, si la relación directa entre las relaciones laborales y el rendimiento de los trabajadores, se evidencian en los operadores turísticos de Santa Marta, siendo éstas un factor clave para el correcto desempeño de sus actividades, esto lo confirman Katz \& Khan (1970), (citados por Rocha, 2005), los cuales definen que “Toda organización crea su propia cultura o clima, con sus propios tabúes, costumbres y estilos. El clima o cultura del sistema refleja tanto las normas y valores del sistema formal como su reinterpretación en el sistema informal". (p.36)

\section{Comportamiento Organizacional}

Es interesante descubrir cómo a través de muchos estudios se ha demostrado que la cultura organiza- 
cional influye directamente en el desempeño laboral, el compromiso y la satisfacción de los trabajadores, ya que cualquier cambio que se le realiza a la cultura es en la búsqueda por aumentar la competitividad de la organización (Palacio, 2012, p.74). Acerca de este tema Robbins (1998) habla explícitamente de la influencia de las culturas fuertes en las organizaciones afirmando que: "Una cultura fuerte evidencia un firme acuerdo entre los miembros sobre lo que representa la organización. Tal unanimidad de propósito favorece la cohesión, la lealtad y el compromiso con la empresa, lo que a su vez, aminora la propensión a que los empleados renuncien". (p.257); lo anterior, es una dinámica creciente de las organizaciones en la actualidad, frente a su estrategia de adaptación a las necesidades de sus empleados para el logro de su fidelidad y compromiso.

Trabajar en equipo para las organizaciones es una necesidad actual, se observa en las definiciones realizadas por De la Cruz \& Hernández (2007, p.24) y Kwantes \& Boglarsky (2007, p.209), que la capacidad de los trabajadores para desarrollar sus labores en equipo tiene una influencia positiva en el logro de objetivos organizacionales, dado que sus actividades parten del principio de cooperación lo que al mismo tiempo influye en que los empleados tengan una fuerte identificación con la organización, generando en ellos la motivación para superar la descripción del trabajo con el fin de mejorar la productividad del mismo.

Por otra parte, en la tendencia del comportamiento organizacional como elemento generador de valor - McGregor (1960) (citado en Chiavenato 2004, pp.136-137) con respecto a este tema, describe que el progreso y desarrollo de la teoría del comportamiento y la motivación contribuyen a establecer parámetros que hacen más práctico el estudio y desarrollo de la Cultura Organizacional y su influencia sobre el rendimiento de las personas en su actividad laboral; McGregor al afirmar que la función de la administración es orientar todas las áreas y recursos hacia mejoramiento de la actividad de la empresa resalta, que la administración no se encarga de crear las condiciones sino de propiciar el ambiente para que las personas desarrollen por si mismas el liderazgo y motivación en su ambiente de trabajo.
Si la cultura organizacional está arraigada a la satisfacción laboral que tenga un empleado en su trabajo, se deduce entonces que está netamente ligada al rendimiento laboral; es así como la socialización de normas de comportamiento formales e informales dentro de la organización también influyen en la creación de una cultura fuerte, que en efecto aumenta la productividad de las personas en las organizaciones, ya que estos aprecian no solo su trabajo sino que reconocen el valor de los procesos en la organización sintiéndose motivados. (De la Hoz \& Mejía, 2002, p.75; Ching Gu, Hoffman, Cao \& Schniederjans, 2013, p.2; Chernyak \& Tziner, 2014, p.4).

Se infiere que los objetivos del comportamiento organizacional se concatenan con los de la organización al describir el modo en el que se conducen las personas y comprender el por qué lo hacen; que le permita establecer parámetros para predecir futuros comportamientos $y$, finalmente, controlar en cierto grado las actividades humanas en pro del logro de las metas organizacionales, afectando directamente su compromiso y satisfacción laboral, cambiando no la cultura sino el comportamiento de las personas. De esta forma, se puede decir que toda persona tiene una participación activa en las organizaciones, por medio de la cual puede alcanzar sus objetivos individuales, pero de igual forma no basta con ser eficiente en el desarrollo de su trabajo sino eficaz en su participación en la organización, para que contribuya a la consecución de los objetivos organizacionales (Drucker, 1994, p.187; Lau \& Ngo, 1996, p.470; Amorós, 2007, p.20; Guillén \& Aduna, 2008, p.52; Wong \& Laschinger, 2012, p.950).

\section{Rendimiento Laboral}

Se encuentra que, para Del Toro, Salazar \& Gómez (2011, p.211), el desempeño laboral es un elemento que varía de un trabajador a otro, como consecuencia de la influencia que ejercen las aptitudes y actitudes de éstos en el desarrollo de las labores realizadas en la empresa, así como inciden sus niveles de capacitación, motivaciones personales, supervisión, en otros factores situacionales inherentes al ambiente de trabajo. En confirmación de dichas suposiciones, se encuentran los aportes realizados por Ruiz, P., Ruiz, C. \& Martínez (2012, p.22) quienes sostienen 
que el simple hecho de tener personal altamente capacitado dentro de la organización y con alto nivel cognitivo, le brinda un valor agregado a la empresa; ya que en algunos ambientes laborales se considera poco usual encontrar personal que realmente posea altas capacidades para desempeñar de manera adecuada una tarea.

Estructurar la cultura organizacional y la forma en la que incide en el rendimiento de las organizaciones, sirve de punto de partida para estudiar el desempeño laboral de los empleados, se utiliza como registro de éxito o fracaso dentro de las organizaciones y elemento que influye en la creación de valores y comportamientos evidenciables en símbolos, lenguajes, ritos difundidos entre los miembros y que resultan favorables o desfavorables en el servicio que ofrecen. En otras palabras, puede que no esté escrita pero se palpa en la forma de hacer las cosas de las organizaciones, puesto que contribuye al aporte de nuevas ideas en beneficio de la competitividad de la empresa. (Pettigrew, 1979, p.570; Azanzaa, Morianob y Molero, 2013, p.46).

Todo lo descrito, pretende establecer las relaciones que se dan entre variables como las condiciones de trabajo, las relaciones laborales y el comportamiento organizacional con el rendimiento laboral de los empleados. La profundización en los aportes teóricos antes expuestos, se hace aún más relevante, en la medida que estos logren demostrar su relación con el rendimiento laboral de los empleados en sus organizaciones, para el caso objeto de estudio, en los operadores turísticos de Santa Marta. Lo anterior, se muestra en los resultados obtenidos.

\section{Metodología}

La investigación sobre la cultura organizacional en el rendimiento laboral de los Operadores Turísticos de Santa Marta, se enmarca en las investigaciones de tipo descriptiva, puesto que al intentar analizar la cultura como factor influyente en el rendimiento laboral, lo que se pretende es puntualizar las variables que caracterizan la cultura organizacional que efectivamente inciden en el comportamiento de los trabajadores y por ende en su rendimiento laboral.

La razón por la cual se seleccionan los 0peradores turísticos es, porque son estas entidades las que facilitan el recorrido turístico de los visitantes que con mayor interés visitan cada día la ciudad de Santa Marta; es por ello que es necesario definir los operadores de Turismo, para conocer el tipo de empresas a las cuales específicamente se realizará el estudio, tomando como base la definición de la Organización Mundial de Turismo en palabras de Valencia (2012, p.3) quien describe que los operadores turísticos: "Son aquellos agentes que participan en la Actividad Turística en calidad de intermediarios entre el consumidor final y el producto turístico".

La obtención de información primaria se generó en encuestas estructuradas realizadas de forma directa a los miembros (trabajadores y directivos) de las empresas turísticas de Santa Marta en una muestra de 27 organizaciones, tomada de una población total de 138 operadores registrados en la ciudad, y de igual forma se realizaron algunas entrevistas a los directivos de dichas instituciones.

Aplicando la fórmula propuesta por Herrera (2012, p.1), para el cálculo de tamaño de muestra cuando el universo es finito, es decir, contable y la variable de tipo categórica, primero debe conocer $N$ (la población), es decir, el número total de empresas; para esto se revisaron los datos estadísticos de COTELCO por el cual para la presente investigación se determinó una población de 138 organizaciones.

La población objeto de estudio está conformada por hoteles, restaurantes y agencias de viajes, lo que conforma el mencionado total de 138 empresas caracterizadas de la siguiente forma: Un total de 75 hoteles en la ciudad, 37 de los cuales se encuentran afiliados a la Asociación Hotelera y Turística de Colombia (COTELCO) ; 53 restaurantes afiliados a la Asociación Colombiana de la Industria Gastronómica (ACODRES); y 10 Agencias de viajes afiliadas al Fondo de Promoción Turística de Santa Marta (FTPS).

La muestra efectiva en la cual se realizó el estudio es de 27 Organizaciones conformada de esta manera: 15 hoteles de los afiliados o no a COTELCO, 10 restaurantes y 2 agencias de Viaje. Esta muestra se obtuvo por el método de muestreo aleatorio simple en el cual se seleccionaron las organizaciones para la posterior recolección de información. 
Se realizó la recolección de los datos a través de encuestas realizadas a los directivos y empleados de las organizaciones en estudio, para la elaboración de dicha herramienta se tomó como base la escala que Rensis Likert por medio del Instrumento para Medir Clima en Organizaciones Colombianas (IMCOC) para medir las diferentes variables en estudio (Pardo, 2000, p.26); ya que esta herramienta se considera apropiada para el análisis y relación de variables porque permite detallar las variables de liderazgo, motivación, comunicación y control por la cual el administrador se apoya en los subordinados para fortalecer la unidad y el potencializar el funcionamiento de las áreas de la organización.

\section{Resultados}

Con base en la información obtenida después de aplicado el instrumento de recolección de datos a los distintos operadores turísticos, se diseñaron los diferentes gráficos de barras, en donde se consolidan los datos correspondientes a las medias de los totales de las encuestas por organización. Del gráfico, se puede comparar con mayor visión y argumentos el desarrollo que tiene la cultura organizacional en relación con el rendimiento de los trabajadores y la productividad de éstos dentro de las empresas. Lo anterior, permitió relacionar las variables de condiciones de trabajo, entorno laboral y comportamiento organizacional con la productividad de la organización.

A continuación se presentan los gráficos obtenidos con sus respectivas interpretaciones.

\section{Condiciones de Trabajo}

\section{- Figura 1.}

Relación Salario Vs. Productividad de los empleados

\section{Salario Vs Productividad}

$$
\square \text { No } \square \mathrm{Si}
$$

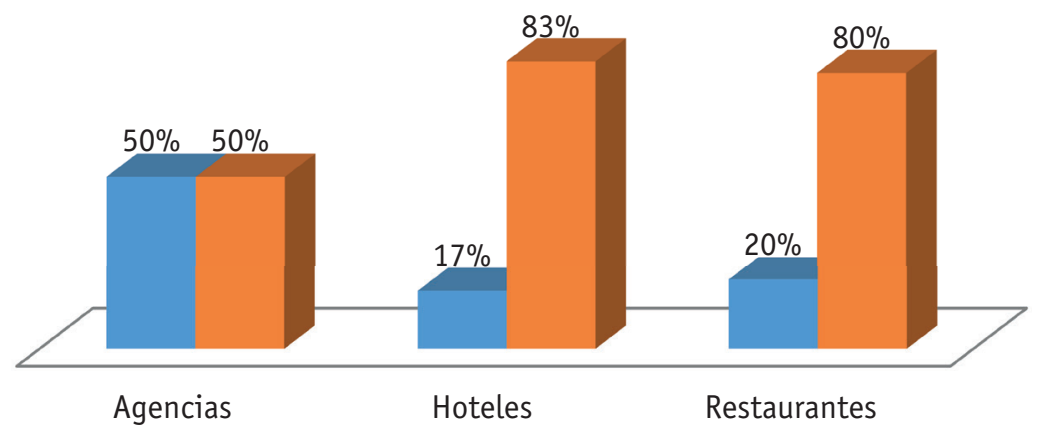

Fuente: Autores. 
La figura 1 muestra que la gran mayoría de los encuestados en los restaurantes y hoteles piensan que existe una directa relación entre el salario devengado y su productividad en la organización; mientras que este pensamiento en las agencias de viajes es dividido, dado que, solo el $50 \%$ considera que evidentemente hay una relación entre las variables confrontadas. Destacando en el caso de los hoteles, quienes obtuvieron el porcentaje más alto $(83 \%)$ en dicha relación, el $42 \%$ de las personas que laboran en estas empresas manifestaron respecto a la variable salario, que ésta no es suficiente para cubrir sus necesidades, mientras que para las personas que laboran en las agencias de viajes y los restaurantes, en su totalidad indican que salario que devengan cubre sus necesidades.

\section{Figura 2.}

\section{Relación de condiciones de trabajo Vs productividad de los empleados}

\section{Condiciones de trabajo Vs Productividad}

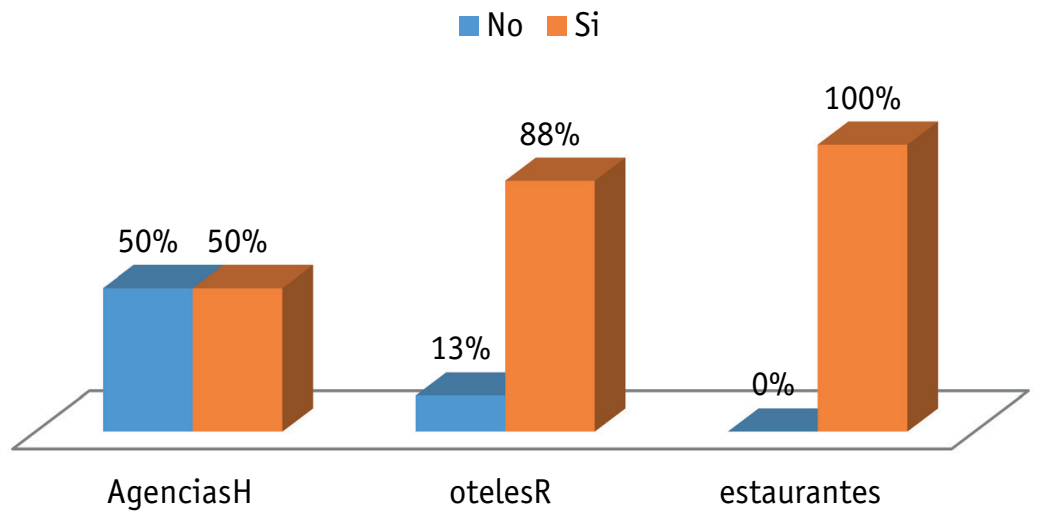

Fuente: Autores.

El gráfico 2 indica que el $88 \%$ y $100 \%$ de las personas que laboran en hoteles y restaurantes respectivamente consideran que las condiciones de trabajo influyen fuertemente en la productividad, en tanto que en las agencias de viajes, solo un $50 \%$ lo considera de esta manera. Enfatizando en el caso de los hoteles, donde la mitad de los encuestados manifestó respecto a las condiciones de seguridad sentirse inseguros en el trabajo, mientras que la otra mitad indico sentirse medianamente seguros; a diferencia de las agencias de viajes y los restaurantes donde las personas exteriorizan seguridad en el trabajo. Cabe mencionar que para gran parte de los encuestados con respecto a las condiciones y elementos físicos en el trabajo, declaran que la empresa les suministra tales condiciones y elementos, en la proporción en que les permite desarrollar cómodamente las actividades diarias de sus funciones. 
Entorno Laboral

fFigura 3.

Variables de entorno laboral: Relaciones laborales Vs productividad

\section{Relaciones Laborales Vs Productividad}

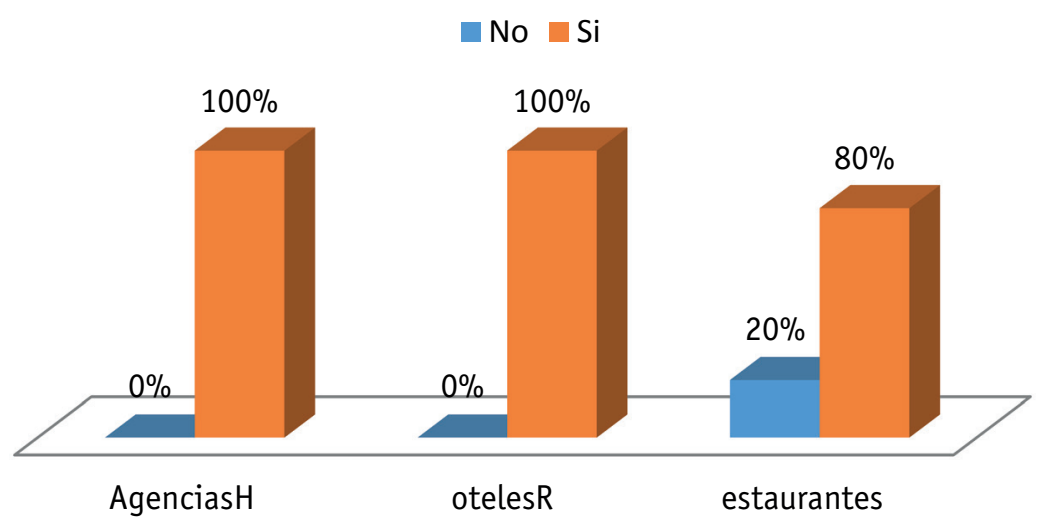

Fuente: Autores.

|Figura 4.

Variable de entorno laboral: Relación con el jefe

\section{Relación con el Jefe}

$\square$ Agencias Hoteles $\square$ Restaurantes

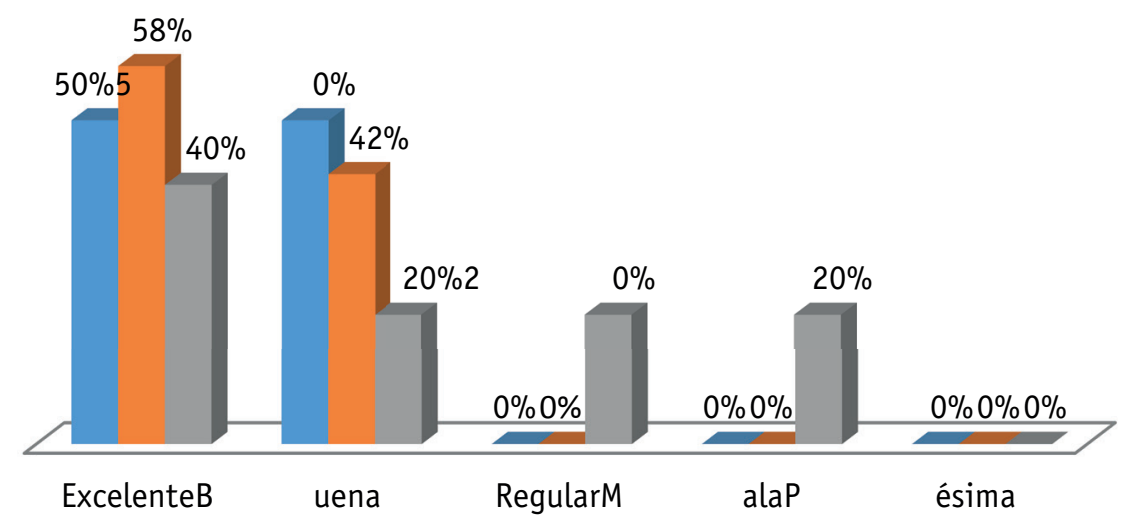

Fuente: Autores. 
No hay duda de que las relaciones laborales inciden directamente en la productividad de la empresa, la mayoría de encuestados (figura 3) pertenecientes a hoteles y agencias de viajes creen en un $100 \%$ tal relación, en tanto que en los restaurantes este porcentaje disminuye ubicándose en un $80 \%$ los que consideran la incidencia de las relaciones sobre la productividad. Dicho resultado que responde a que la mayoría de las personas sondeadas manifiestan encontrar siempre y algunas veces apoyo en sus compañeros, además piensan que al presentarse algún conflicto encuentran apoyo y alternativas para llegar a alguna solución.

La investigación muestra (figura 4) que, la relación con los jefes para la mayoría de los encuestados en los hoteles, agencias de viajes y restaurantes es calificada entre excelente y buena; destacando particularmente a los restaurantes donde el $40 \%$ de los colaboradores consideran que dicha relación no merece ser calificada como buena.

- Figura 5.

Variables del entorno laboral: Comunicación con el jefe Vs productividad

\section{Comunicación jefes Vs Productividad}

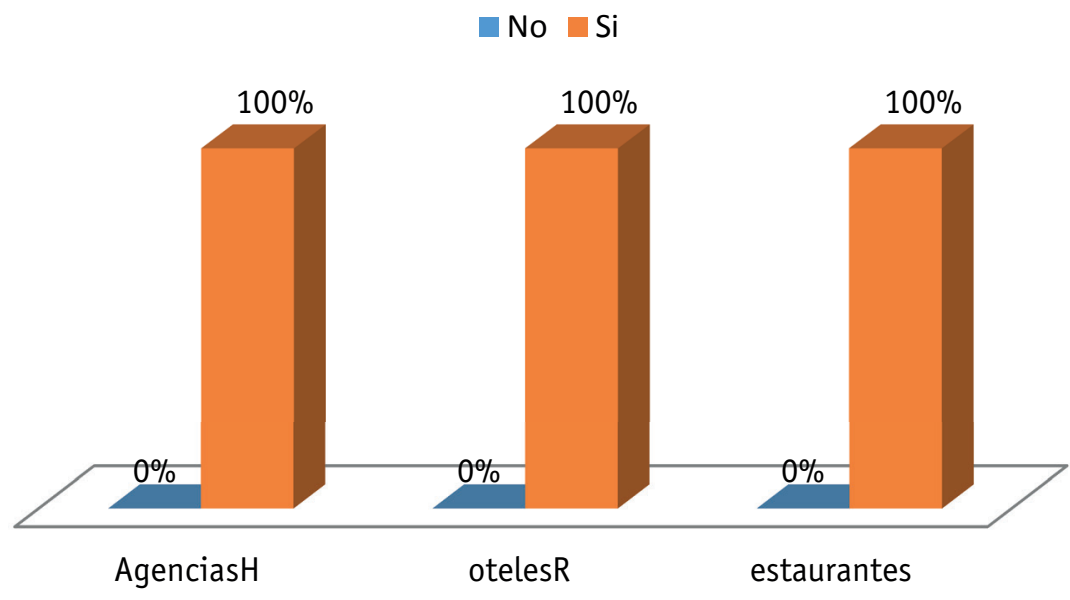

Fuente: Autores.

La figura 5 evidencia que la totalidad de los encuestados en restaurantes, las agencias y los hoteles piensa que existe una relación directa entre la comunicación que se tiene en la organización con la productividad de ellos y la del hotel, dado que para la mayoría de los encuestados es de vital importancia establecer y desarrollar una comunicación fluida y precisa con las altas directivas, para que de esta forma se puedan alcanzar de una mejor manera las metas organizacionales. 


\section{Comportamiento Organizacional}

Figura 6.

Variable del comportamiento organizacional: Liderazgo

\section{Liderazgo}

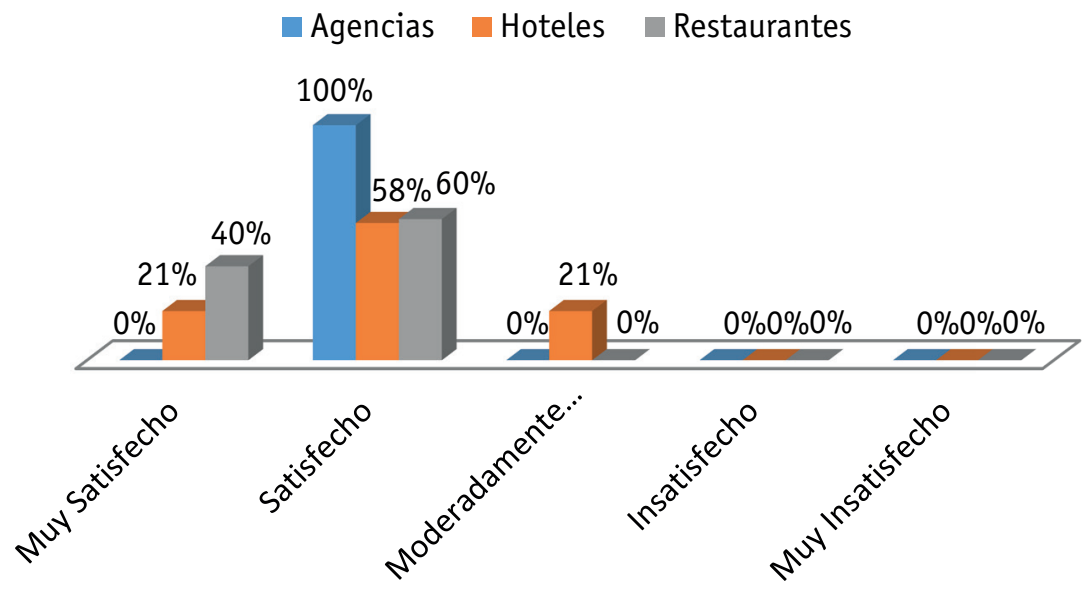

Figura 7.

Variable del comportamiento organizacional: Trabajo en equipo

\section{Trabajo en Equipo}

$\square$ Agencias $\quad$ Hoteles Restaurantes

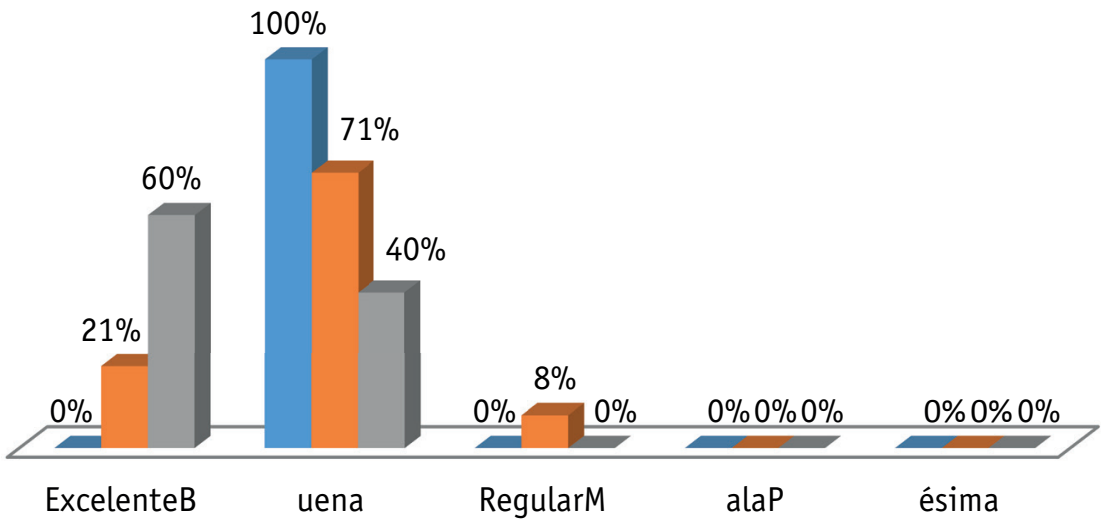

Fuente: Autores. 
La investigación mostró que la gran mayoría de los encuestados (figura 6) en restaurantes, las agencias y los hoteles piensa que sus empresas les otorgan libertad para ejercer cierto liderazgo en su puesto de trabajo y en la organización, por lo cual se encuentran muy satisfechos y satisfechos.

La investigación mostró que un alto porcentaje de los encuestados (figura 7) en restaurantes, las agencias y los hoteles piensan que sus empresas fomentan el trabajo en equipo en la organización como estrategia organizacional que les permite interconectar cada dependencia y de esta forma lograr los objetivos en conjunto; además indican que el grado de satisfacción por esta estrategia es excelente y buena.

La investigación mostró que la gran mayoría de los encuestados (figura 8) de los operadores turísticos piensan que existe una relación directa el comportamiento organizacional que se tiene en la empresa, con la productividad de ellos y la de los restaurantes, agencias de viajes y hoteles.

Figura 8.

Relación del comportamiento organizacional Vs productividad de los trabajadores

\section{Comportamiento organizacional Vs Productividad}

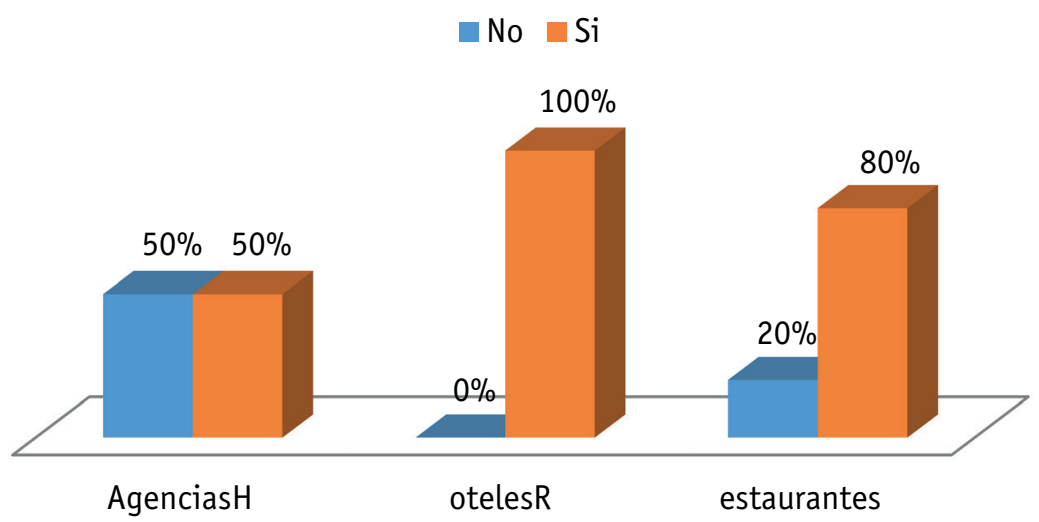

Fuente: Autores.

\section{Discusión Y Conclusión}

Dado que en la totalidad de las gráficas se hace la interpretación y discusión de los resultados obtenidos, se resaltan los hallazgos más importantes:
- La gran mayoría de los encuestados manifiesta que las buenas relaciones interpersonales, la comunicación organizacional, las relaciones con sus jefes y compañeros, tienen relación directa con su productividad y la de las empresas, sin 
Jennifer Tatiana Ortiz Segrera, Alexander Daza Corredor y Carlos Labarcés Ballestas

embargo, es relevante destacar que un $40 \%$ de los colaboradores en los restaurantes consideran que las relaciones con los jefes no se desarrollan de la mejor manera.

- Los empleados sienten apoyo en la resolución de conflictos cuando se presentan en el desarrollo de su trabajo, enfatizando que el $100 \%$ de las agencias se encuentra satisfecha con el apoyo que recibe; sin embargo, es representativo que en otros momentos durante el desarrollo de las labores, los empleados de los operadores turísticos, sienten el apoyo de sus compañeros solo algunas veces; lo que afecta su productividad en la organización.

- Las condiciones laborales, equipos y seguridad que ofrecen los empleadores a sus empleados, hace que estos se sientan muy satisfechos, lo anterior, redunda en la productividad de ellos y la organización.

- El otorgar los empleadores a sus empleados condiciones que les permita tomar decisiones, ejercer su liderazgo, el trabajo en equipo y la auto-superación, redunda en su productividad y la de la organización.

- Los trabajadores de los operadores turísticos sienten en gran medida que se les brinda cierto grado de libertad en la toma de decisiones concernientes a su área de trabajo, casi siempre o algunas veces, permitiéndole aumentar su productividad.

\section{Referencias Bibliográficas}

Açıkgöz, A. \& Günsel, A. (2011). The effects of organizational climate on team innovativeness. Procedia Social and Behavioral Sciences 24. pp. 920-92. Recuperado el 7 de Marzo de 2013 de: http://www.sciencedirect.com/science/ article/pii/S1877042811016302

Amorós, E. (2007). Capítulo 1. ¿Qué es el comportamiento organizacional? Comportamiento Organizacional. ISBN-13: 978-84-690-4674-6. Escuela de Economía USAT, Perú. Recuperado el 15 de Octubre de 2013 de: http://books.google.es/books?hl=es\&lr=\&id=uRfl1b4 $4 B j E C \& 0 i=$ fnd\&pg=PA2\&dq=teoria + de + comportamien to+organizacional\&ots=XqZhFm9IWH\&sig=PrtXGQ4_ BXydJT_mLb0RGKX1dM\#v=onepage\&q=teoria\%20de\%20 comportamiento $\% 20$ organizacional\&f=true

Azanzaa, G., Morianob, J. \& Molero, F. (2013). Authentic leadership and organizational culture as drivers of employees' job satisfaction. Journal of work and Organizatioanl Psychology 29(2013). pp. 45-50. Recuperado el 7 de abril de 2014 de: http://www.sciencedirect.com/science/article/pii/ S1576596213700070

Calderón, G. \& Serna, H. (2009). Relaciones entre recursos humanos y cultura organizacional. Un estudio empírico. Tesis inédita de Pregrado, Universidad Nacional de Colombia, Manizales, Colombia. Recuperado el 10 de Marzo de 2013 de: http://portalweb.ucatolica.edu.co/easyWeb2/ files/23_2739_v12n2-art9.pdf

Chernyak, L. \& Tziner, A. (2014). Relationships between counterproductive work behavior, perceived justice and climate, occupational status, and leader-member exchange. Journal of work and organizational psychology 30(2014). pp. 1-12. Recuperado el 7 de abril de 2014 de: http://apps.elsevier.es/ watermark/ctl_servlet?_f=10\&pident_articulo=90283388\&pident_usuario $=0 \&$ pcontactid $=\&$ pident_revista $=370 \&$ ty $=5 \&$ ac cion=L\&origen=jwop\%20\&web=jwop.elsevier.es\&lan=en\&fic hero $=370 v 30$ n01a90283388pdf001.pdf

Chiavenato, I. (2004). Administración de Recursos Humanos. Bogotá, Colombia: Editorial McGraw-Hill Interamericana S.A.

Ching Gu, V., Hoffman, J., Cao, Q. \& Schniederjans, M. (2013). The effects of organizational culture and environmental pressures on IT project performance: A moderation perspective. International Journal of Project Management $x x$ (2013). pp. xxx-xxx. Recuperado el 19 de Enero de 2014 de: http://www.sciencedirect.com/science/article/pii/ S0263786313001774

Chiang, F. \& Birtch, T. (2011). Reward climate and its impact on service quality orientation and employee attitudes. International Journal of Hospitality Management 30 (2011). pp. 3-9. Recuperado el 15 de Febrero de 2014 de: http://www.sciencedirect.com/science/article/pii/ S0278431910000290

De la Cruz, J. \& Hernández, J. (2007). Diagnóstico de la cultura y el clima organizacional en la empresa Aceites S.A del municipio de El Retén, Magdalena. (Tesis inédita de Pregrado). Universidad del Magdalena, Santa Marta, Magdalena.

De la Hoz, G. \& Mejía, M. (2002). La motivación como factor fundamental en el mejoramiento de la satisfacción laboral, clima laboral y la productividad de los trabajadores de la finca María de la Esperanza ubicada en la Zona Bananera del departamento del Magdalena. (Tesis inédita de pregrado). Universidad del Magdalena, Santa Marta, Magdalena.

Del Toro, J., Salazar, M. \& Gómez, J. (2011). Clima organizacional, satisfacción laboral y su relación con el desempeño laboral en trabajadores de un PYME de servicios de ingeniería. Revista CLIO América 5 (10). pp. 204-227. 
Doherty, A. \& MacIntosh, E. (2009). The influence of organizational culture on job satisfaction and intention to leave. Sport Management Review 13 (2010). pp. 106-117. Recuperado el 05 de Marzo de 2013 de: http://www.sciencedirect.com/science/article/pii/S1441352309000515\#

Drucker, P. (1994). Capitulo 26 Cultura Corporativa: Emplearla, no perderla. Gerencia para El Futuro El Decenio de los 90 y Más Allá. pp. 185-190. Colombia: Editorial Carvajal S.A.

Gálvez, E. \& García, D. (2011). Cultura organizacional y rendimiento de las Mipymes de mediana y alta tecnología: Un estudio empírico en Cali, Colombia. Cuadernos Administración Bogotá (Colombia), 24 (42). pp. 125-145. Recuperado el 28 de Febrero de 2014 de: http://revistas. javeriana.edu.co/index.php/cuadernos_admon/article/ view/1749/1105

González, J. \& Parra, C. (2008). Caracterización de la cultura organizacional Clima organizacional, motivación, liderazgo y satisfacción de las pequeñas empresas del Valle de Sugamuxi y su incidencia en el espíritu empresarial. Pensamiento \& gestión, 25. pp. 40-57. Universidad del Norte, Barranquilla, Colombia. Recuperado el 28 de Febrero de 2014 de: http:// rcientificas.uninorte.edu.co/index.php/pensamiento/ article/viewFile/3195/2159

Guerrero, Y. \& Navarro, B. (2003). Análisis y diagnóstico del clima organizacional en la planta de personal administrativo del a Universidad del Magdalena de Santa Marta D.T.C.H durante el año 2003.( Tesis inédita de pregrado). Universidad del Magdalena, Santa Marta, Magdalena.

Guillén, I. \& Aduna, A. (2008). La influencia de la cultura y del estilo de gestión sobre el clima organizacional. Estudio de caso de la mediana empresa en la delegación Iztapalapa. Estudios Gerenciales 24 (2008). pp. 47-64. Recuperado el 20 de febrero de 2014 de: http://www.sciencedirect.com/ science/article/pii/S0123592308700317

Herrera, M. (2012). Fórmula para cálculo de la muestra poblaciones finitas. Recuperado el 15 de Noviembre de 2012 de: http://investigacionpediahr.files.wordpress.com/2011/01/ formula-para-cc3a1lculo-de-la-muestra-poblacionesfinitas-var-categorica.pdf

Jarratt, D. \& O'Neill, G. (2002). The Effect of Organisational Culture on Business-to-Business Relationship Management Practice and Performance. Australasian Marketing Journal 10 (3). Recuperado el 7 de Marzo de 2013 de: http://www.sciencedirect.com/science/article/pii/ S1441358202701560\#

Kahya, E. (2007). The effects of job characteristics and working conditions on job performance. International Journal of industrial Ergonomics 37 (2007). pp. 515-523. Recuperado el 5 de marzo de 2013 de: http://www.sciencedirect.com/ science/article/pii/S0169814107000418\#

Kahya, E. (2009). The effects of job performance on effectiveness. International Journal of Industrial Ergonomics 39 (2009). pp. 96-104. Recuperado el 5 de marzo de 2013 de: http://www.sciencedirect.com/science/article/pii/ S0169814108001133\#

Kwantes, C. \& Boglarsky, C. (2007). Perceptions of Organizational Culture, Leadership Effectiveness and Personal Effectiveness Across Six Countries. Journal of International Management 13 (2007). pp 204-230. Recuperado el 2 de Noviembre de 2012 de: http://www.sciencedirect.com/science/article/ pii/S1075425307000282\#

Lau, C. \& Ngo, H. (1996). One Country Many Cultures: Organizational Cultures of Firms of Different Country Origins. International Bussines Review Vol. 5, No. 5. pp. 469-486. Recuperado el 2 de Noviembre de 2012 de: http://www. sciencedirect.com/science/article/pii/0969593196000224

Méndez, C. (2006). Clima organizacional en Colombia el IMCOC: Un método de análisis para su intervención. Colección Lecciones Facultad de Administración. Bogotá. Centro Editorial Rosarista. Recuperado el 14 de Agosto de 2012 de: http://mutis.urosario.edu.co/bitstream/10336/914/1/ Clima\%20organizacional_.pdf

Morales, L. (2010). Caracterización de la cultura organizacional en empresas colombianas. (Tesis inédita de pregrado). Universidad del Rosario, Bogotá, Colombia. Recuperado el 28 de 0ctubre de 2012 de: http://repository.urosario. edu.co/bitstream/handle/10336/1789/1032375920-2010. pdf;jsessionid=A88FC4F1067443A642E5372975AD1F3F?se quence $=3$

Palacio, J. (2012). Capitulo 3: Hoja de Ruta para generar valor en la Gestión Humana. La Paz Laboral: ¿Costo o inversión?. pp. 49-78. Medellín, Colombia: Fondo Editorial Ascort.

Pardo, M. (2000). Identificación del Perfil de Clima organizacional de información gerencial Ltda.. Bogotá. Universidad de la Sabana. Recuperado el 15 de Noviembre de 2012 de: http://intellectum.unisabana.edu.co:8080/jspui/ bitstream/10818/2428/1/005.pdf

Pettigrew, M. (1979). On studyng Organizational Cultures. Administrative Science Quarterly 24 (12). pp. 570-581. Recuperado el 7 de marzo de 2013 de: http://www.jstor. org/discover/10.2307/2392363?uid=3737808\&uid=2\&uid $=4 \&$ sid $=21103636318307$

Robbins, S. (1998). Capitulo 16: La Cultura Organizacional. Fundamentos de Comportamiento Organizacional. pp. 253268. México: Prentice-Hall Hispanoamericana, S.A. 
Rocha, M. (2005). La cultura organizacional en Pemex gas y petroquímica básica. (Tesís inédita de maestría). Instituto Politécnico, Nacional, México. Recuperado el 5 de marzo de 2013 de: http://tesis.bnct.ipn.mx/dspace/bitstre am/123456789/1937/1/1412_2005_ESCA-ST_MAESTRIA_ rocha_rodriguez_marioluis.pdf

Ruiz, P., Ruiz, C. \& Martinez, R. (2012). Cultura organizacional ética y Generación de valor sostenible. Investigaciones Europeas de Dirección y Economía de la Empresa 18 (1). pp. 17-31. Universidad de Castilla, La Mancha. Recuperado el 29 de enero del 2014 de: http://apps.elsevier.es/watermark/ ctl_servlet?_f=10\&pident_articulo=90134236\&pident_ usuario $=0 \&$ pcontactid $=\&$ pident_revista $=345 \&$ ty $=136$

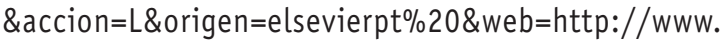
elsevierciencia.es\&lan=es\&fichero=345v18n01a9013423 6pdf001.pdf

Tavares, S. (2004). Cultura organizacional e postura estratégica da empresa. Revista Ibero - Americana De Estratégia, 3(1). pp. 63-77. São Paulo: UNINOVE Recuperado el 1 de Marzo de 2014 de: http://search.proquest.com/docview/90065 0660? accountid $=43960$

Tsui, A., Zhang, Z., Wang, H., Xin, K. \& Wu, J. (2006). Unpacking the relationship between CEO leadership behavior and organizational culture. The Leadership Quartely 17 (2006). pp 113-137. Recuperado el 7 de Marzo de 2013 de: http://www.sciencedirect.com/science/article/pii/ S1048984305001712
Turró, A., Urbano, D. \& Peris, M. (2013). Culture and innovation: The moderating effect of cultural values on corporate entrepreneurship. Technological Forecasting \& Social Change (2013). Recuperado el 29 de Enero de 2014 de: http://www.sciencedirect.com/science/article/pii/ S004016251300262X

Tziner, A. \& Sharoni, G. (2014). Organizational citizenship behavior, organizational justice, job stress, and work-family conflict: Examination of their interrelationships whit respondents from non-Western culture. Journal of Work and Organizational Psychology 30(2014). Pp. 35-42. Recuperado el 7 de abril de 2014 de: http://apps. elsevier.es/watermark/ctl_servlet?_f $=10 \&$ pident_articulo $=90284019 \&$ pident_usuario $=0 \&$ pcontactid $=\&$ pident_revista $=370 \&$ ty $=123 \&$ accion $=$ L\&origen $=j w o p \% 20$ \&web=jwop.elsevier.es\&lan=en\&fichero=370v30n01a902 84019pdf001.pdf

Valencia, J. (2012). Boletín turístico. Recuperado el 02 de Septiembre de 2013 de: http://www.boletin-turistico.com/ diccionarioturismo/Diccionario-1/0/page, $3 /$

Wong, C. \& Laschinger, H. (2012). Authentic leadership, performance, and job satisfaction: the mediating role of empowerment. Journal of Advanced Nursing. 69(2012). pp. 947-959. Recuperado el 7 de abril de 2014 de: http://onlinelibrary.wiley.com/doi/10.1111/j.1365-2648.2012.06089.x/pdf 